\title{
«Est enim eloquentia valde necessaria prædicationi»: Bernardino da Siena e la sermocinatio
}

"Est enim eloquentia valde necessaria prædicationi »: Bernardino

de Sienne et la sermocinatio

"Est enim eloquentia valde necessaria prædicationi": Bernardino of Siena and the Sermocinatio

\section{Valentina Berardini}

\section{OpenEdition}

\section{Journals}

\section{Edizione digitale}

URL: https://journals.openedition.org/cei/5785

DOI: 10.4000/cei.5785

ISSN: 2260-779X

\section{Editore}

UGA Éditions/Université Grenoble Alpes

\section{Edizione cartacea}

ISBN: 978-2-37747-159-1

ISSN: 1770-9571

\section{Notizia bibliografica digitale}

Valentina Berardini, ««Est enim eloquentia valde necessaria prædicationi»: Bernardino da Siena e la sermocinatio», Cahiers d'études italiennes [Online], 29 | 2019, online dal 30 septembre 2019. consultato il 21 septembre 2021. URL: http://journals.openedition.org/cei/5785; DOI: https://doi.org/ 10.4000/cei.5785

Questo documento è stato generato automaticamente il 21 septembre 2021. 


\title{
«Est enim eloquentia valde necessaria prædicationi»: Bernardino da Siena e la sermocinatio
}

\author{
«Est enim eloquentia valde necessaria prædicationi »: Bernardino \\ de Sienne et la sermocinatio \\ "Est enim eloquentia valde necessaria prædicationi": Bernardino of Siena \\ and the Sermocinatio
}

Valentina Berardini

\section{Introduzione}

1 A partire dagli anni Ottanta del secolo scorso, gli studi sui sermoni medievali si sono arricchiti di un nuovo filone di indagine, volto all'analisi dei testi e dei manoscritti attraverso cui le prediche sono state trasmesse. Da allora i sermoni sono stati oggetto di numerose e diversificate ricerche che ne hanno messo in risalto il valore di 'atti comunicativi', basati sulla diffusione di una 'parola' destinata ad essere pronunciata e udita. L'interesse per lo studio dei testi ha quindi condotto all'analisi di sermoni inediti e alla scoperta delle note stenografiche (reportationes) registrate dagli ascoltatori, durante l'esecuzione stessa del sermone.

Individuato il valore della predicazione quale fenomeno essenziale alla conoscenza del Medioevo e della sua cultura, i nuovi studi ne hanno messo in risalto le connessioni con le altre manifestazioni del vivere sociale e civile, quali ad esempio il pensiero teologico e politico, l'arte e il teatro ${ }^{1}$. A tale proposito è importante rilevare l'influenza esercitata dagli Ordini Mendicanti in ambito architettonico in cui si assiste al nascere di strutture ecclesiastiche utili a favorire una migliore ricezione della voce del predicatore ${ }^{2}$. Elemento centrale per la diffusione della predica era il pulpito che progressivamente 
viene spostato verso la parte centrale della navata, probabilmente proprio a seguito dell'influenza esercitata dagli Ordini Mendicanti. La sua disposizione poteva variare anche sulla base di valutazioni acustiche ma è importante sottolineare che il pulpito non era semplicemente il luogo deputato alla divulgazione della predica ma anche strumento rafforzativo del messaggio evangelico, potenziato dal repertorio iconografico del pulpito ${ }^{3}$.

3 Proprio in virtù del rapporto fra la predicazione e le altre manifestazioni del vivere sociale e civile, un recente filone di indagine si è soffermato sulla natura 'spettacolare' del sermone e, avvalendosi delle riflessioni prodotte nell'ambito della performance theory, si interroga sugli strumenti utili alla ricostruzione di un evento per sua natura effimero e irripetibile.

4 A tal proposito, in un articolo ${ }^{4}$ dedicato all'analisi del rapporto fra la teoria della predicazione e le modalità con cui la stessa venne realizzata, Beverly Kienzle rilevava le difficoltà connesse con lo studio dei sermoni. Infatti, l'assenza di testimonianze in grado di restituire l'evento nella sua totalità, ovvero dotato di tutti quegli elementi tipici delle comunicazioni orali (gesti, intonazione della voce, espressione facciale, emozione, reazione del pubblico), costringe gli studiosi a interrogarsi sui metodi utili a tentare di ricostruire il fenomeno della predicazione, ricercando nei documenti scritti le tracce dell'originaria oralità.

5 Muovendo da tali considerazioni e seguendo la traccia proposta da Beverly Kienzle, ho dedicato la mia ricerca all'esame dei rapporti fra predicazione e teatro, definendo un modello di analisi volto a rintracciare all'interno dei sermoni gli indicatori di una teatralità implicita ${ }^{5}$.

6 In particolare, la mia ricerca si rivolge ai sermoni divulgati da Bernardino da Siena nei suoi cicli di predicazione tenuti a Firenze e a Siena, rispettivamente nel 1424, nel 1425 e nel $1427^{6}$.

7 La scelta del corpus omiletico di Bernardino da Siena non è casuale e deriva, da un lato, dal carattere straordinario ed originale della sua predicazione, dall'altro dalla possibilità di servirsi di reportationes che registrano i procedimenti retorici cui Bernardino fece ricorso. D'altra parte lo studio del carattere teatrale dei testi bernardiniani si inserisce in una lunga tradizione di studi cui si cerca di dare un contributo originale offrendo un'analisi sistematica dei sermoni, volta a individuare fenomeni, elementi e procedimenti responsabili di un'espressione mimetica e drammatica, capace di fare ricorso a codici comunicativi diversi da quello verbale.

La lettura diretta dei testi mira ad individuare una serie di elementi che possono essere considerati 'indicatori', segnali, di una modalità espositiva ampiamente coincidente con il codice comunicativo tipico del mondo teatrale.

Alla luce delle caratteristiche assunte dalle manifestazioni teatrali nel Medioevo, possiamo considerare teatrale o potenzialmente tale un testo che si serve di strumenti in grado di favorire il passaggio da un discorso teorico e dottrinale a un atto comunicativo che si avvicina alla messa in scena e alla mimesi. La presenza di tali strumenti suggerisce, infatti, la sovrapposizione del codice comunicativo visuale e dialogico, tipico del teatro, al codice comunicativo verbale, caratteristico di ogni comunicazione orale. 
10 La teatralità implicita identifica quindi quegli elementi in grado di interrompere il tono didattico del sermone attraverso il ricorso a immagini e formule comunicative tipiche di altri ambiti e, in particolare, del teatro.

11 A tali fenomeni, individuati in base alla capacità di alleggerire il peso teorico del sermone e di tradurne visivamente il contenuto, ho dato il nome di 'indicatori di teatralità implicita' in quanto 'spie' della componente drammatica insita nella predica. Tali indicatori, così individuati, sono stati suddivisi in due classi corrispondenti a due livelli di drammatizzazione del discorso evangelico: il teatro di sfondo e il teatro-mimo.

12 Al primo livello - quello del teatro di sfondo - appartengono le forme espressive che suggerendo la presenza di un interlocutore, implicano una comunicazione del tipo iotu, trasformando il monologo in dialogo. Pur prendendo parte alla comunicazione il tu rimane silenzioso, o, meglio, si esprime per bocca dello stesso Bernardino che ne riferisce, interpretandoli, pensieri e parole. In questo modo la dialogicità si arricchisce di una componente mimetica che conferisce carattere teatrale all'intera comunicazione. Rientrano in questo gruppo anche esclamazioni e formule onomatopeiche, che conferiscono espressività al discorso del predicatore.

13 Tali indicatori concorrono alla creazione di quello che ho definito 'teatro di sfondo' ovvero il primo livello di drammatizzazione del testo in quanto costituisce la situazione comunicativa di base sulla quale si innestano le spiegazioni teologiche e le esposizioni evangeliche.

14 Al secondo livello - il teatro mimo - corrispondono invece episodi di tipo narrativo che grazie all'uso di particolari procedimenti, vengono sottoposti a un processo più $o$ meno forte di drammatizzazione. Trasformati da semplici narrazioni in scene, gli episodi appartenenti a tale livello testimoniano delle qualità mimetiche del predicatore, al contempo narratore e attore delle scene rappresentate. Rientrano, a titolo esemplificativo, in tale gruppo gli exempla che hanno un ruolo fondamentale per la divulgazione e la memorizzazione del messaggio evangelico.

15 Un particolare tipo di indicatore che rientra nel primo livello di drammatizzazione del testo è rappresentato dalla sermocinatio della quale ora si parlerà in modo più approfondito.

\section{Sermocinatio}

La sermocinatio, dal latino sermocinari, «conversare, discutere», è una figura retorica che consiste nel riportare in forma diretta enunciati altrui. In questo modo, all'interno del discorso pronunciato dal locutore principale trovano spazio parole e frasi proprie di altri locutori, diversi dal soggetto parlante.

17 Le origini della sermocinatio sono antiche ed è probabile che tale figura retorica sia stata

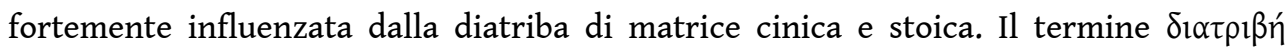
significava, originariamente, «conversazione»; in seguito venne utilizzato per indicare i discorsi divulgati dai filosofi al popolo e, come tali, caratterizzati da una particolare forma espositiva che riprendeva i modi argomentativi popolari ${ }^{7}$.

18 Le potenzialità insite nella sermocinatio erano note da tempo, fin dalle origini della predicazione cristiana e prima di Bernardino da Siena, Giordano da Pisa ${ }^{8}$ vi aveva fatto ricorso per scongiurare la monotonia del monologo. Infatti, simulando un dialogo fra due interlocutori, tale figura retorica consente di interrompere il monologo 
dell'oratore conferendo al sermone un tono più espressivo. La sermocinatio, dunque, conferisce al sermone un forte impianto dialogico che stimola costantemente la reazione e l'attenzione degli ascoltatori, coinvolti in qualità di protagonisti all'interno del discorso pronunciato dal predicatore. Il valore della sermocinatio risulta particolarmente notevole soprattutto nei casi in cui sia importante mettere in evidenza i sentimenti e gli stati d'animo dell'interlocutore, sostituendo la forma narrativa con una forma più dinamica $a^{9}$ stabilendo un contatto intimo fra predicatore e pubblico ${ }^{10}$.

Date tali caratteristiche, la sermocinatio risultava uno strumento utilissimo per Bernardino da Siena che mirava ad enunciare precetti morali capaci di guidare il fedele nella vita quotidiana. Per tale ragione doveva fare ricorso non solo a un nuovo progetto tematico ma anche e soprattutto a una nuova strategia comunicativa capace di collocare il sermone allo stesso livello degli ascoltatori. Era, quindi, molto importante trasmettere un messaggio che fosse non solo comprensibile e memorizzabile ma soprattutto capace di coinvolgere gli ascoltatori così da garantirsene e assicurarsene l'attenzione per tutta la durata della predica.

Le varianti sotto cui può presentarsi la sermocinatio sono diverse ma possono essere ricondotte a tre forme principali ${ }^{11}$ : discorso senza dialogo, dialogo, monologo contenente interrogativi deliberativi e non necessariamente dotato della struttura

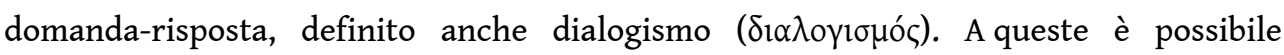
aggiungere una quarta forma che costituisce, in realtà, una variante del secondo tipo, ovvero della "sermocinatio dialogica», e nota anche come percontatio; questa "consiste nella finzione [...] di un dialogo dell'oratore o con il suo avversario o con il pubblico; l'oratore ripete interrogativamente una finta domanda o una finta osservazione dell'avversario e aggiunge quindi una risposta antitetica» ${ }^{12}$.

21 Caratteristiche delle tre forme principali della sermocinatio sono: a) il discorso diretto riferito ad altrui e riportato fedelmente dal predicatore, b) l'alternanza di battute fra due o più interlocutori e c) la formulazione di domande rivolte dall'oratore a sé stesso. Tali procedimenti prevedono quindi il ricorso a una formula espositiva che ben si presta ad una resa di tipo mimetico basata sull'imitazione delle espressioni tipiche dell'interlocutore del cui discorso il locutore principale si fa interprete e portavoce.

Applicando i procedimenti tipici della sermocinatio, Bernardino da Siena ricorre ampiamente al dialogo, senza però tralasciare altri procedimenti che si collocano in un punto intermedio tra la prima e la seconda forma ovvero fra discorso senza dialogo e dialogo puro.

23 Tali procedimenti sono stati qui ripartiti in quattro categorie principali: 1) simulazione di domande provenienti dal pubblico; 2) mimesi di espressioni proprie dei suoi ascoltatori; 3) esplicitazione dei pensieri dei fedeli; 4) imitazione di risposte o obiezioni provenienti dagli spettatori. A queste è poi da aggiungere la categoria del dialogo che rappresenta la cifra caratteristica dei sermoni bernardiniani.

\subsection{Simulazione di domande provenienti dal pubblico}

24 Nel caso della simulazione di domande provenienti dal pubblico, Bernardino formula una domanda fingendo che questa provenga da qualcuno dei suoi ascoltatori; la domanda, dunque, non è rivolta al pubblico bensì al predicatore. 
Ad esempio, nella predica recitata il 27 marzo 1424 a Firenze («Di cosa si è obbligati a rendere»), Bernardino narra l'exemplum di una donna molto bella la quale era solita partecipare alle feste per farsi ammirare. Apparentemente il comportamento della donna non arreca nessun danno ma in realtà ruba l'anima di chi la guarda. Individuata la colpa, Bernardino offre al proprio pubblico un modo per potersi pentire e, per farlo, introduce una domanda che sembra pronunciata da qualcuno dei suoi ascoltatori:

Ora allo ammendare. Come debbo io fare, frate Bernardino? Dirottelo. Vesti per lo contrario. Ristora, vesti onestamente te e tue figliuole e tuoi figliuoli, e anco meno che non richiede el tuo stato. Se ài la coda, mozzala, e va' co' panni onesti. Se t'adornavi troppo, non t'adornare punto $[. . .]^{13}$.

Il discorso del santo è interrotto da una domanda che sembra provenire da qualcuno fra il pubblico, forse una delle donne riconosciutasi colpevole dello stesso peccato. Alla domanda, Bernardino risponde con tono deciso e assertivo («Dirottelo») e indica la soluzione per rimediare al peccato commesso e seguire la via virtuosa. L'introduzione della domanda è, quindi, funzionale a spiegare in che modo è possibile adottare un comportamento giusto rinunciando agli atteggiamenti assunti in precedenza. In questo modo non solo il discorso si fa più vivace ma soprattutto Bernardino fa accedere alla scena i suoi ascoltatori, rendendoli protagonisti e coinvolgendoli nel suo discorso. È molto probabile che, nel formulare la domanda, Bernardino abbia mutato il proprio tono di voce per rendere evidente che a porla era persona diversa dal predicatore. Per concludere, è importante rilevare come il passaggio dal monologo del predicatore alla domanda proveniente dal pubblico avvenga in maniera molto naturale e spontanea, come accadrebbe in un reale scambio dialogico fra due persone impegnate in una conversazione in cui le frasi dell'una si sovrappongono a quelle dell'altra.

In altri casi la domanda proveniente dal pubblico, e formulata dal predicatore, scaturisce da un dubbio sorto negli ascoltatori in conseguenza di quanto esposto poco prima da Bernardino; è quanto avviene, ad esempio, nel sermone recitato l's marzo 1424, dedicato al tema della confessione. Avendo sottolineato l'importanza e il valore della confessione, Bernardino afferma:

Mostrasi che cosa è confessione e come di somma necessità, e quello secondo isprendore del quarto lume. E potresti tu muovere in questo un dubbio e dire: «Ove truovi tu che io debba confessare e miei peccati?». E dicotelo ${ }^{14}$.

Rispetto all'esempio precedente, dove la domanda sembrava scaturire spontaneamente dal pubblico, qui la mediazione da parte di Bernardino è molto più evidente e lo scambio dialogico meno diretto. Tuttavia, gli effetti della sermocinatio non vengono meno e rimane intatta la sua capacità di vivacizzare la predica rompendo il monologo del predicatore.

È possibile trovare un esempio simile nella predica del 16 marzo 1425, in cui Bernardino affronta il tema dei «ma' contratti»; come è noto, la pratica dell'usura era molto diffusa e i predicatori tentavano di reprimerla, eleggendola spesso ad argomento centrale dei propri sermoni. Avendo mostrato il peccato commesso prestando il denaro a usura, Bernardino esplicita il dubbio sorto, a suo avviso, nelle menti dei suoi ascoltatori:

Uno dubio ti vego nascere nella mente. Dirai tu: «S'io presto una casa a costui, o una bottega, o una vigna, o uno cavallo a cotanto il dì, o a cotanto il mese, o a cotanto l'anno, e dimmi ch'egli è lecito, e possogli pigliare le pigioni, e i fitti, e le vetture de' ronzini; adunque perché non posso io, per lo simile, appigionare o allogare i miei danari e torne quel prezzo che rendessono in sul podere, o in sulla casa, o in sul cavallo poi ch'egli si gode e aopera i miei danari?». Tu lo colori molto bene ${ }^{15}$. 
eno dall'ascoltatore non è del tutto erroneo e tuttavia si tratta di un escamotage per aggirare il divieto imposto relativamente alla pratica dell'usura; per tale ragione, il predicatore, dopo aver apprezzato il tentativo da parte del fedele di scagionarsi dal peccato commesso, rileva la nullità del suo ragionamento soffermandosi sulle differenze che intercorrono fra il prestare a usura e l'affitto di un bene di altro genere. Analogamente a quanto rilevato in precedenza, Bernardino, simulando il dubbio sorto negli ascoltatori, chiarisce il concetto senza appesantire il discorso e mostrandosi anzi vicino ai modi di pensare e sentire dei suoi fedeli.

Bernardino ricorre alla sermocinatio anche nel ciclo del 1427 introducendo, a volte, forme più raffinate che rafforzano l'impianto dialogico del sermone. Nella predica recitata il 24 settembre e dedicata al tema della «mercatanzia», il Santo accusa i religiosi che si dedicano al commercio, in quanto intraprendono un'attività che compete esclusivamente agli uomini secolari. Fissato tale principio, Bernardino specifica che, a differenza del fornicare, da considerarsi sempre azione illecita, il mercatare assume carattere più o meno peccaminoso a seconda della persona che lo svolge. A tale scopo, si serve di una domanda per specificare in quale misura ai religiosi è concesso svolgere attività commerciale:

El fornicare è inlecito, ma il mercatare è lecito. Ma costui che è fatto religioso, non gli è lecito più di mercatare. - $\mathrm{O}$ non è lecito a prete a vendare il suo grano, avendone d'avanzo, per avere de' denari, per comparare di quelle cose che elli ha di bisogno? - Sì. - E a compararne? - Dico che sì. - Quello che si può fare, ode Pavolo scrivendo a Timoteo, capitolo III: «Nemo militans Deo se implicat in opere seculari». Né voi secolari dovete dare uffizio a religiosi; né anco i religiosi nol debbano accettare, né cercare, né esercitare ${ }^{16}$.

Grazie al susseguirsi delle domande il discorso si trasforma in un dialogo reale, immediato, diretto. Rispetto agli esempi precedenti la tecnica oratoria di Bernardino è più raffinata: il predicatore non si limita a simulare la domanda proveniente dal pubblico ma fornisce anche la sua risposta oscillando continuamente tra il ruolo di predicatore e il ruolo di spettatore.

sermone in oggetto offre l'occasione per verificare l'evoluzione dell'arte omiletica bernardiniana; infatti nel ciclo del 1424 Bernardino aveva affrontato lo stesso tema e, analogamente a quanto affermato a Siena, aveva sottolineato l'impossibilità per i religiosi di intraprendere attività di tipo commerciale. Tuttavia la modalità di esposizione, pur vivace, non ricorre alle domande evidenziate nel passo citato e il sermone si presenta quindi in una veste diversa:

La prima si è della persona che la fa, ché a ogni persona non è lecito fare mercatanzia. A' preti, a' frati, a' monaci, gli abati, i prelati, a monache e generalmente a niuno regolato non è lecito il fare niuna mercatanzia, sotto pena di peccato mortale. [...]

Possono bene vendere le loro ricolte, olio, vino, grano, biade e altre cose, ma per farne mercatanzia, no, in niuno modo. È tanto proibito a loro il fare la mercatanzia, per lecita che la sia in sé, quanto è proibito a' secolari il prestare a usura. E piglia questa regola generale, che al pre' o al regolato che di vile o povero stato sia venuto a gran riccheza - come dice santo Girolamo - egli è peggio e peggiore che niuna bestia pestifera. E messer san Pagolo il testifica a Timoteo nelle sue Pistole ove ammunisce che niuno regolato s'impacci in mercatanzie ${ }^{17}$.

Dal confronto risulta chiaro come Bernardino non muti lo schema della predica e ricorra, in entrambi i casi, alle auctoritates per dare valore al suo argomento. Le differenze maggiori emergono, piuttosto, a livello narrativo laddove la predica del 1424 
assume un tono impersonale e didascalico, mentre in quella del 1427 è più evidente l'urgenza comunicativa e la volontà di dar vita a un dialogo capace di tenere desta l'attenzione del pubblico.

\subsection{Mimesi di espressioni proprie dei suoi ascoltatori}

La seconda variante della sermocinatio è rappresentata dalla mimesi di espressioni proprie del pubblico le cui formule espositive sono molto vicine a quelle analizzate per i dubbi del pubblico ma differiscono in quanto si presentano con un semplice intento dichiarativo.

Così ad esempio nella predica recitata il 21 marzo 1425 a Firenze, Bernardino da Siena sintetizza le affermazioni più comuni dei giovani che si dispongono a "pigliar moglie»:

Or cominciamo prima da' giovani che vogliono moglie, e intanto voi, donne, vi pensate. E parliamo in sul sodo. Dirai tu, giovane: «Io la vorrei la moglie savia; io la vorrei onesta; io la vorrei vergine; io la vorrei che non avesse mai avuto amore a niuno uomo; vorre' la che non fusse ghiotta né ubriaca; non vorrei fusse una gracchiuola né contenditrice; vorre' la paziente che non s'adirasse d'ogni cosa; vorre' la ubidiente di quello le comandassi; vorrei ch'ella non fusse una gallessa; vorrei essere il gallo io; vorrei ch'ella non fusse troppo lussuriosa; non vorrei ch'ella fusse una maliarda; vorrei avere una moglie che io la potessi contentare de' vestimenti per non diventare povero a sua cagione; vorre'la tutta costumata e che sapessi essere con ogni gente; vorrei fusse fattiva e non una mentacatta, sapessi fare d'ogni minima cosa di casa, e anche mi sapesse guardare e accrescere la mia masserizia; la vorrei virtudiosa e che temesse Iddio» ${ }^{18}$.

Il continuo ripetersi del verbo vorrei conferisce al brano un tono sostenuto contribuendo al raggiungimento di un ritmo incalzante, scandito dal susseguirsi delle diverse qualità ricercate nelle donne. Inoltre, il pronome di prima persona «io» sembra riferibile a soggetti diversi, il cui modo di parlare e di esprimersi è molto probabilmente imitato dal predicatore che così sottolinea l'eterogeneità delle esigenze manifestate.

In altre occasioni, la mimesi di espressioni proprie del pubblico serve a riprendere $\mathrm{i}$ fedeli, imitando alcune delle scuse addotte a giustificazioni di peccati commessi o di cattivi comportamenti adottati. Così, per esempio, nel sermone recitato il 10 marzo 1424 a Firenze e dedicato al tema della confessione difettosa Bernardino mostra alcune delle giustificazioni portate dai fedeli per aver commesso qualche peccato:

Nella quale figura e ammaestramento ci dà ad intendere che in niuno modo si vuole sé scusare, a Dio imputare, il prossimo incolpare, il dimonio appiccare, ché molti peccati si commettono prima in dire bugie, e non avere amore né riverenza a Dio, e non amare il prossimo. E aggiugnesi male a male e peccasi di nuovo mortalmente, e non se' confessato, come molti, dicono: «Orbene, io ò fatto il tale contratto, io mi sono adirato in casa co' mia figliuoli, colla mia famiglia, colla mia donna, ma non si può fare altro; egli è usanza di dire bugie nel vendere e nel comprare; o io feci cattiva misura o cattivo peso, ma usasi così, ma altrimenti io ne perderei ecc.; o il diavolo mi fece cadere in questo peccato; o veramente l'amprensione delle pianete m'ànno dato questa inferenza che io sono superbo; o io sono lussurioso, o simile nature, io non posso fare altro; o el tale m'indusse al male; ò il tale peccato che per me medesimo non l'arei fatto...» eccetera ${ }^{19}$.

Analogamente, nella predica recitata il 19 aprile 1424 a Firenze, Bernardino affronta il tema della comunione e polemizza contro coloro che si astengono dal sacramento con la scusa di temere di cadere nuovamente nel peccato: 
Pognamo che tu abbi preso el corpo di Gesù Cristo nella santa pasqua, e poi, per fragilità, ricaggi nel peccato, meglio ti rileverai da quel peccato, avendolo preso, che se tu non l'avessi preso. [...] Le mille volte cadessi, mille volte ti rilieva. Non dire: «Io non mi voglio comunicare perch'io ricadrei a mano a mano in peccato» ${ }^{20}$.

In altri casi, quale è quello del sermone recitato a Firenze l'8 marzo 1425 in cui si analizzano gli aspetti della mala vita, la mimesi di espressioni proprie del pubblico non si serve di elementi demarcativi essendo inserita all'interno del discorso del predicatore come naturale commento a quanto espresso da Bernardino da Siena:

La terza è del vestimento pomposo. Dice che era vestito di vestimento di bisso e di porpora. I troppi vestimenti fanno a' vostri mariti fare ogni male per contentarvi. Di fuori, dice, vestimento di seta; di dentro di pannilini sottili. Io stupisco, fra Bernardino, come niuno confessore n'assolve niuna che porti tanti vestimenti o tanti ornamenti superflui. Dottori, debbono contrapporsi a' vestimenti pomposi e con troppo studio fatti ${ }^{21}$.

L'osservazione dell'ascoltatore, stupito per la tolleranza dei confessori nei confronti della vanità femminile, è incastonata all'interno delle parole del predicatore e la sua alterità non è segnalata da alcun elemento demarcativo, fatta eccezione per quell'inciso - «fra Bernardino» - che segnala la provenienza dell'allocuzione da persona diversa dal predicatore.

41 A volte la mimesi può limitarsi a una sola frase, come avviene nella predica recitata a Siena il 18 agosto in cui Bernardino imita le parole che una madre è solita rivolgere al proprio figlio quando questi non si comporta nel modo adatto: «Sai, come fa la madre quando ella ha il fanciullino, che ella il minaccia e dice: "Se tu non fai così, io te ne pagarò" " ${ }^{22}$. È plausibile pensare che in questo caso Bernardino abbia imitato sia il tono della voce sia l'atteggiamento della madre nei confronti del figlio, magari accompagnando l'espressione con un gesto di tipo ammonitivo; appare quindi chiaro che la predilezione per il discorso diretto era funzionale, fra l'altro, a una resa di tipo drammatico capace di coinvolgere tanto la modulazione della voce quanto la gestione del corpo, piegato alle esigenze comunicative.

Infine, giova qui rilevare la funzione fatica del Sai iniziale che predispone ad un atteggiamento confidenziale fra predicatore e ascoltatori.

\subsection{Esplicitazione dei pensieri dei fedeli}

43 La terza variante della sermocinatio è rappresentata dal pensiero del pubblico esplicitato. In questo caso la mimesi poteva riguardare sia le espressioni più comuni sia i pensieri e le reazioni degli ascoltatori come avviene nelle prediche dell'11 e del 16 marzo 1424 a Firenze. Il passaggio dal discorso del predicatore alla mimesi del pensiero dell'ascoltatore è diretto e privo di elementi introduttivi:

Quando altri è uscito del mare cioè dei pericoli, allora si conosce Iddio che prima nella nave e nel mare non lo conoscevi. E allora pensi e ripensi. O di quanti mali e di quanti peccati sono campato e uscito per la grazia di Dio e non per mio merito né per mia bontà! ${ }^{23}$.

Ma in su' peccati carnali non vi dimorare punto. Passatene leggermente ché vi potresti incappare, e pensa e ripensa in generale e passa via. Ispezialmente pensati i 
peccati fatti el dì passato: così parlai al tale; così dissi la tale bugia, feci la tale cosa di peccato ${ }^{24}$.

Analogamente a quanto rilevato in precedenza, Bernardino passa agilmente dal discorso in prima persona, che lo coinvolge in qualità di predicatore, alla mimesi delle parole dell'ascoltatore di cui riproduce il pensiero.

Diversamente accade nella predica recitata il 13 febbraio 1425 (dedicata al tema del peccato) dove il passaggio dal discorso di Bernardino a quello dei fedeli è marcato in maniera più netta attraverso il ricorso a quegli elementi demarcativi rilevati in precedenza a proposito della mimesi delle espressioni del pubblico:

Se tu ài l'amore al vivere bene, come tu di', fuggi le cagioni che ti potrebbono impedire a vivere bene, come tu desideri; ma se tu non fuggi le cagioni, è segno che tu non disideri di bene vivere, e poi v'è poca discrezione. Che se dicessi in te medesimo: «quale è meglio, o a fare a modo mio confidandomi di me, o a fare a modo di messer Domeneddio che dice ch'io fugga»; diresti Iddio ne sa più di te, e seguiteresti il suo consiglio, e non andresti drieto a seguire la tua prosunzione ${ }^{25}$.

Gli esempi non mancano nel ciclo senese e infatti nel sermone recitato il 26 settembre, Bernardino invita i suoi fedeli a considerare le conseguenze del mancato rispetto dei comandamenti divini e individua quali potrebbero essere le rassicurazioni rivolte dai peccatori a sé stessi rappresentandole sotto forma di discorso diretto: «Vedendo ciò che Idio ti comandò che tu facesse e non l'hai fatto, che dirai tu in te medesimo? Forse tu dirai: "Egli mi comandò cosa che io non la potevo fare" ${ }^{26}$.

La stessa formula si ritrova nella predica del 17 agosto 1427 in cui si legge: «E quando tu andarai a fare uno contratto, tu vi pensarai prima dicendo: "Che disse frate Bernardino? Elli mi disse così e così: questo è male, non si convien fare: questo è bene, questo vo' fare" $»^{27}$.

Nelle espressioni citate la forza mimetica è in parte attenuata rispetto agli esempi appartenenti alle altre categorie analizzate; tuttavia, in quanto unico 'attore' sulla scena, è plausibile pensare che Bernardino fosse costretto ad assumere particolari accorgimenti fonici per segnalare ai propri uditori i suoi due ruoli di predicatore e imitatore di espressioni altrui.

\subsection{Imitazione di risposte o obiezioni provenienti dagli spettatori}

L'ultimo dei procedimenti individuati afferisce ancora una volta alla prima delle forme in cui può manifestarsi la sermocinatio ovvero il discorso senza dialogo.

Le espressioni racchiuse all'interno di tale gruppo presentano caratteristiche simili a molti dei fenomeni analizzati finora ma sono state distinte da quelli in quanto si configurano o come risposte a una domanda posta da Bernardino da Siena o come obiezioni ad un'affermazione da questi formulata.

Nel primo caso, l'espressione del pubblico è sempre mediata dall'intervento di Bernardino che, pur mettendo in atto un procedimento mimetico, ricorre a elementi che segnalano l'imitazione realizzata dal predicatore; in altri termini alla domanda non segue direttamente la risposta bensì l'esposizione, in forma diretta, della replica del pubblico. Tale procedimento risulta più chiaro se si prende ad esempio la predica recitata il 17 marzo 1424 a Firenze: «Quale cosa si debba più amare o il grande bene $o$ il minore bene? Risponderai: "El maggior bene" e dirai el vero»" ${ }^{28}$. La risposta del pubblico 
non è presentata in forma diretta, al contrario il verbo «Risponderai» evidenzia la mimesi in atto.

51 Analogamente, nel sermone del 13 aprile dello stesso anno si legge: «A che fare portate voi tutte le cose e le fogge che portano le meritrici? Rispondera': "Per piacere al mio marito, acciò che io li piaccia più, e che non caggi in sodomia" ${ }^{29}$.

Dagli esempi si ha come l'impressione che Bernardino voglia segnalare la sua capacità di 'indovinare' la risposta più plausibile, dimostrando, così, di conoscere perfettamente la psicologia dei suoi fedeli. Tale procedimento se da un lato testimonia delle qualità $\mathrm{di}$ acuto osservatore proprie del santo, dall'altro vanifica, anche se solo in parte, l'effetto teatrale altrove raggiunto grazie all'impiego di formule linguistiche più dirette, capaci di nascondere la mediazione messa in atto dal predicatore nel riproporre frasi altrui.

È possibile ravvisare lo stesso meccanismo anche nei casi in cui Bernardino si fa portavoce delle obiezioni sollevate dai suoi fedeli, formulate, forse, sulla base delle reazioni percepite nel pubblico e portate sulla scena per dar voce ai suoi ascoltatori in modo da coinvolgerli attivamente nella predica, trasformandoli in protagonisti attivi.

Per esempio, trattando del tema della confessione, nella predica del 10 marzo 1424 , Bernardino esorta i suoi fedeli a rivolgersi ai preti che abbiano la licenza del confessare e non siano stati scomunicati: «E piglia questa regola generale, che ogni reciso e preciso dalla chiesa di Dio e scomunicato, non vale sua confessione $»^{30}$. Immediatamente Bernardino immagina l'obiezione dei suoi ascoltatori: «Ma tu potrai dire: "O che so io che sia scomunicato, o ch'egli abbia autorità, o ch'egli sia reciso o preciso dalla chiesa!" Dicoti che, se tu non lo sai, che ti basta la coscienza» ${ }^{31}$. L'inserimento della formula introduttiva del discorso diretto («Ma tu potrai dire»), a scapito della sua inserzione senza ricorso ad alcun elemento demarcativo, segnala la mimesi in atto rendendo evidente che l'autore della frase è il predicatore, immedesimatosi in uno dei suoi ascoltatori.

Analoghe formulazioni linguistiche ricorrono anche nel ciclo senese in cui il Santo adotta gli stessi meccanismi per mostrare le obiezioni mosse dal pubblico alle sue raccomandazioni e ai suoi insegnamenti. Infatti, molto spesso accade che Bernardino si serva di tali meccanismi per presentare sulla scena le scuse addotte dai fedeli per non svolgere le azioni loro raccomandate durante la predica; in questo modo egli era in grado di vanificare subito le reticenze dei suoi ascoltatori dimostrandone la nullità e non lasciando loro possibilità di sottrarsi ai doveri del buon cristiano. Per esempio, in occasione della predica tenuta il 21 settembre, Bernardino riporta le principali scuse addotte dai fedeli che praticano l'usura a giustificazione del peccato commesso: «Dice colui: - Oh, io lassarò poi, che elli si dia per li povari, quando io morrò, o per maritare fanciulle, o fare chiese o spedali, o altre opere piatose a gloria di Dio!» ${ }^{32}$; immediata è la risposta del predicatore che non consente al fedele di mettere al riparo la propria coscienza:

E io ti dico che come tu consenti di prestare a usura, subito hai fatto contra al comandamento di Dio. Idio te l'ha negato, e però ti dico che per niuno modo puoi prestare a usura, e tu non li debbi dare né consiglio né vigore, né con parole né con fatti, che mai si presti ${ }^{33}$.

Dunque non vi è possibilità di scampo: prestare a usura, anche se accompagnato da un gesto positivo quale può essere la devoluzione del denaro per opere di bene, rimane comunque un'attività peccaminosa nei confronti della quale non esistono attenuanti di alcun genere. 


\subsection{Dialogo}

La creazione di dialoghi fittizi è uno dei fenomeni più tipici e caratteristici della predicazione bernardiniana e appartiene alla seconda variante della sermocinatio. In questa sede saranno presi in esame solo i dialoghi in cui agiscono il predicatore e una persona del pubblico; saranno invece tralasciati tutti quei dialoghi che vedono coinvolti i personaggi dei tanti exempla che arricchiscono la predicazione bernardiniana. Come si è già detto, molti dialoghi scaturiscono dallo sviluppo di alcuni dei procedimenti tipici della sermocinatio in forma di discorso senza dialogo.

Si prenda, ad esempio, il sermone recitato a Firenze il 18 aprile 1424, dedicato al tema del perdono: dopo aver narrato un exemplum, Bernardino imita la reazione di uno dei suoi ascoltatori: «Dirà alcuno indemoniato: "Or bene non dirò el Pater nostro acciò che io non invuochi contro a me" $\rangle^{34}$. L'espressione è una mimesi dell'espressione del pubblico e, al contempo, un'obiezione a quanto precedentemente espresso circa l'importanza di conoscere e recitare la preghiera principale della liturgia cristiana. Tuttavia, a differenza degli esempi esposti in precedenza, alla mimesi non segue la normale ripresa della spiegazione del predicatore; al contrario, questi reagisce e risponde, prendendo avvio proprio dalla dichiarazione del suo ipotetico ascoltatore:

E io ti dico: Se tu non lo dirai tu non ti salverai mai, e se non lo di' e vadi alla messa, quando el prete el dice, prega Iddio della tua maladizione. È meno male che tu non vada alla messa che tu non dica el Pater nostro ${ }^{35}$.

Ne consegue un andamento piuttosto colloquiale in cui emerge chiaramente l'impianto dialogico che coinvolge in una conversazione 'spontanea' predicatore e interlocutore. La mimesi ha subito quindi uno sviluppo ulteriore consentendo la creazione di una battuta di dialogo che dona movimento e vivacità al sermone.

In altri casi, il dialogo scaturisce dalle domande rivolte dal predicatore ai suoi fedeli; le risposte, presentate come provenienti dal pubblico, sono in realtà pronunciate dallo stesso Bernardino. Ad esempio, nel sermone del 24 settembre 1427, per dimostrare l'insaziabilità degli avari il santo ricorre, in primo luogo, a un'auctoritas, «E però è detto nello Ecclesiastico al V capitolo: "Avarus non satiabitur pecunia. L'avaro non si sazia però de la pecunia"; quanta più n'ha, più ne disidera» ${ }^{36}$; in seguito, Bernardino costruisce un dialogo fittizio che ha come interlocutore uno dei suoi ascoltatori:

Voliamo vedere s'io dico il vero? Or proviallo. O avaro, che vorresti tu? - Io vorrei dieci mila fiorini: s'io avesse dieci mila fiorini, io mi credarei star bene. - Or tèlli. Ha'gli? - Sì. - Oltre. Che n'hai fatti? - Oh, io gli spesi; io ne voglio più. Egli se n'è andato uno mio mezzaiuolo che io gli avevo prestati cento fiorini; io n'ho messi in bestiame; egli mi bisognò cinquanta fiorini per aconciare una casa; egli me ne bisognano più. - Oltre. Quanti ne vuoi? - Io ne vorrei almeno almeno quindici mila. (Già vedi che gli è cresciuto l'animo.) Oltre: togli. Che ne farai? Ha'lo avisato? - Sì. Io ho allato a la mia una casa che mi s'affarebbe molto bene; e così è una pocissione, che mi tramezza fra due ch'io n'ho: s'io la potesse avere, non sarebbe persona che mi potesse far danno; elle sarebbero insieme insieme. - E subito o in questo $o$ in quello egli spende tutti, e anco s'ingegna d'averne più. - Io vorrei più denari. $-\mathrm{O}$ a che te ne bisogna tanti? - Oh, s'io n'avessi un pochi più, per certo ch'io no ne cercarei poi più! - Oltre. Quanti ne vuoi? - Io ne vorrei almeno venticinque mila. $-O$ che ne faresti di tanti? - Oh, che ne farei? Egli è una fortezza in uno luogo che mi s'affarebbe molto; e anco vorrei da ogni porta una pocissione; io so' schifo de la nebbia; se la nebbia fusse da una porta, e io andarei all'altra dove non fusse la nebbia ${ }^{37}$. 
lettura del passo riportato appare evidente l'abilità di Bernardino nel sapersi abilmente destreggiare tra il ruolo di predicatore e il ruolo di ascoltatore; gli scambi di battute sono rapidi, veloci, e conferiscono all'intero passo una struttura dialogica vicina a quella propria degli sketches teatrali. D'altra parte, l'uso del verbo vedere, in apertura del passo, suggerisce proprio l'intenzione di dare vita a una comunicazione basata sul codice visivo, capace di conferire concretezza e realismo al discorso del predicatore. È come se, terminata l'esposizione teorica suffragata dal ricorso all'auctoritas, Bernardino decidesse di 'alzare il sipario' consentendo ai suoi spettatori di vedere con i propri occhi la realizzazione pratica della tesi esposta. Considerato il carattere dialogico e mimetico del brano, rimane difficile immaginare un'interpretazione priva di quei riferimenti gestuali necessari a caratterizzare l'identità dei due interlocutori e a conferire il giusto grado di realismo all'intera scena. Il riferimento a una situazione concreta (il comprare una casa in grado di soddisfare le esigenze sempre nuove e diverse dell'avaro) permette di ancorare il discorso a un riferimento comune, proprio della realtà quotidiana cui il pubblico appartiene, e perciò in grado di fissarsi nella mente e nella memoria degli ascoltatori. cresciuto l'animo.)» - a seguito della seconda richiesta dell'avaro, relativa alla somma di quindicimila fiorini; sembra trattarsi di un 'a parte', destinato a essere udito solo ed esclusivamente dal pubblico esterno alla scena in atto. Non sembra eccessivo attribuirgli valore di ammiccamento rivolto dal predicatore ai suoi ascoltatori a seguito del quale si determina una sorta di complicità da cui resta escluso l'avaro, ignaro della chiosa svolta in relazione alla sua affermazione; di conseguenza, la scena si complica in quanto tale espressione, necessaria a sottolineare il modo di agire e pensare tipico dell'avaro, determina, anche se solo per un momento, la sospensione della mimesi. In altre parole è come se Bernardino strizzasse l'occhio ai suoi ascoltatori per rendere loro evidente il tranello con il quale egli è in grado di smascherare l'insaziabilità del suo interlocutore e, dunque, la validità della tesi proposta in precedenza. Inoltre, in questo modo risulta ancor più evidente l'abilità con la quale Bernardino entra in scena o se ne ritrae, alternando il ruolo di predicatore / oratore a quelli di predicatore / attore e predicatore / interlocutore.

61 A volte l'intenzione di procedere secondo gli schemi tipici di una normale conversazione è evidente fin dalla formula di apertura del passo, introdotto dai verbi 'dire' o 'rispondere' coniugati alla seconda persona del modo imperativo («dimmi»; «rispondi»). In altri termini, il predicatore chiama direttamente in causa il suo pubblico, obbligandolo a prendere parte al discorso in atto; l'atto comunicativo, dunque, da monologico diviene dialogico, sia pure solo apparentemente. Si prenda ad esempio il sermone recitato il 4 aprile 1424 in cui Bernardino tenta di convincere i suoi ascoltatori a liberarsi di tutti quegli strumenti con i quali si è compiuto un danno nei confronti di altri uomini o di Dio. Per raggiungere il suo scopo, il predicatore prospetta al suo pubblico una situazione reale nella quale ognuno potrebbe immedesimarsi e tutto il suo discorso è intessuto di proposizioni interrogative:

Rispondi. Se ti trovassi in casa una ispada o una lancia colla quale fusse stato morto tuo padre, che ne faresti tu? Dimmelo. Or dirai: «Se potrò della lancia o della spada ne farò cento pezzi». Or non sarebbe el meglio a fare di quella lancia qualche cosa per casa, o una stanga o altro e di quella spada farne fare coltellini? «Mai no, dirai tu, perché io non mi voglio vedere innanzi quella cosa ch'è suta cagione di tormi el padre mio». E ài ragione, e così si debba fare. Adunque perché vuo' tu, indemoniato, 
che quelle cose che sono sute strumento di dare el male contro a Dio, e contro a te,

e contro al prossimo tuo si vendino o s'aoperino in altro ${ }^{38}$.

In questo caso l'allocuzione diretta all'interlocutore è stata sviluppata ulteriormente fino ad arrivare alla creazione di un vero e proprio dialogo con un'alternanza ben calibrata di battute che scandiscono i «turni di parola, secondo una tecnica che risente del linguaggio scolastico e soprattutto della tecnica dialogica della qucestio» ${ }^{39}$.

Rispetto all'esempio precedente, l'intervento del predicatore è più evidente in quanto segnalato dalla presenza di formule demarcative («or dirai...», «[...] dirai tu...») ma non per questo viene meno l'effetto dinamico e vivace conferito al testo dall'inserzione di un dialogo.

È importante in questa sede rilevare un esempio di dialogo molto particolare in quanto testimone della capacità creativa e mimetica bernardiniana.

\section{Conclusione}

Sulla base dell'analisi condotta è possibile osservare che lo scopo primario del ricorso alla sermocinatio da parte di Bernardino da Siena è la rottura del monologo del predicatore al fine di conferire movimento e vivacità all'esposizione. Tale espediente risulta particolarmente utile nel momento in cui il predicatore si trova ad esporre argomenti difficili e per i quali è importante ricorrere ad una forma espositiva in grado di mantenere viva l'attenzione degli ascoltatori e di tradurre in termini semplici concetti teologici complessi.

Grazie all'impiego delle differenti forme proprie della figura retorica in oggetto, il Santo riesce a dare vita a una comunicazione di tipo teatrale in cui la mimesi delle espressioni degli interlocutori svolge un ruolo essenziale ai fini della drammatizzazione del testo. Infatti, riportando frasi, dubbi, domande e pensieri dei suoi ascoltatori, Bernardino dà vita a una rappresentazione che lo coinvolge nel duplice ruolo di predicatore e spettatore. Ricorrendo a una metafora teatrale, si potrebbe dire che Bernardino si serve del suo interlocutore come se fosse una 'spalla' e in questo modo approfondisce il discorso senza risultare noioso. Le domande dell'ipotetico ascoltatore introducono spiegazioni e precisazioni che, se fossero state presentate secondo $i$ moduli espositivi tradizionali, avrebbero certamente appesantito il tono del discorso, favorendo la disattenzione del pubblico e il conseguente fallimento degli scopi del messaggio evangelico. Inoltre, l'esplicitazione delle reazioni più comuni al suo messaggio evangelico consente a Bernardino di sfumare la portata teologica del messaggio, peraltro mai del tutto abbandonata grazie alle numerose citazioni scritturali e al continuo riferimento alle auctoritates, e di avvicinare il sermone alle esigenze e alla vita dei suoi ascoltatori. In questo modo egli riduce la tradizionale distanza fra predicatore e pubblico dichiarandosi perfettamente consapevole delle difficoltà incontrate nel tentativo di rispettare i comandamenti divini; pur dimostrandosi in molti casi severo nei confronti del suo pubblico, cui non risparmia rimproveri e minacce, tuttavia sembra proporsi come predicatore giunto con lo scopo di svelare i mezzi per poter raggiungere la salvezza eterna piuttosto che come censore e castigatore della condotta di vita umana.

Inoltre, l'attenzione che Bernardino rivolge alle ipotetiche obiezioni dei suoi ascoltatori rivela l'impegno a confezionare sermoni adatti alle esigenze e alle aspettative del pubblico. Infatti, l'obiezione riprodotta dal predicatore svolge la funzione di abbassare 
il registro linguistico e stilistico del sermone, introducendo il ragionamento / dubbio dell'interlocutore per spiegare in termini più semplici concetti teologici complessi.

D'altra parte, il ricorso alla sermocinatio si inscrive perfettamente all'interno dell'ars omiletica bernardiniana quale teorizzata dallo stesso Bernardino e che individua nello stile «chiarozzo chiarozzo» ${ }^{40}$ la sua cifra distintiva. Infatti, secondo quanto esposto all'inizio del ciclo senese, il predicatore deve, in primo luogo, tradurre i concetti teologici più complessi in termini semplici, chiari e tangibili. A tale fine, la sermocinatio svolge un ruolo importantissimo nel diminuire la distanza fra predicatore e pubblico consentendo al primo di farsi interprete dei pensieri e delle esigenze dei suoi ascoltatori e, al secondo, di accedere al discorso evangelico trovandone attuazione concreta nella vita quotidiana.

\section{BIBLIOGRAFIA}

ALLEGRI Luigi, Teatro e spettacolo nel Medievo, Roma-Bari, Laterza, 1988.

ARASSE Daniel, Saint Bernardin de Sienne. Entre dévotion et culture : fonctions de l'image religieuse au $X V^{e}$ siècle, Paris, Hazan, 2014.

ARDUINI Stefano e DAMIANI Matteo, Dizionario di Retorica, Covilhā, Labcom Books, 2010.

BAXANDALL Michael, Painting and Experience in Fifteenth Century Italy: A Primer in the Social History of Pictorial Style, Oxford, Oxford University Press, 1988.

BeN-ARYeH DeBBy Nirit, The Renaissance Pulpit. Art and Preaching in Tuscany, 1400-1550, Turnhout, Brepols, Late Medieval and Early Modern Studies, vol. 6, 2007.

BERNARDINO DA SIENA, Le prediche volgari, a cura di C. Cannarozzi, Firenze, Tip. Pacinotti, 1934.

BERnARdino DA SIENA, Le prediche volgari, a cura di C. Cannarozzi, Pistoia, Tip. Pacinotti, 1940.

Bernardino Da Siena, Prediche volgari sul Campo di Siena, 1427, a cura di C. Delcorno, Milano, Rusconi, 1989.

CADEI Antonio, È possibile scrivere una storia dell'architettura mendicante? Appunti per l'area padanoveneta, in Tommaso da Modena e il suo tempo, Atti del Convegno Internazionale di Studi per il VI Centenario della morte (Treviso, 31 agosto-3 settembre 1979), Treviso, Comitato per le celebrazioni, 1980, pp. 337-362.

CASAGRANDE Carla e VECCHIO Silvana, L'interdizione del giullare nel vocabolario clericale del XII secolo, in Il contributo alla drammaturgia italiana delle origini, Atti del II Convegno di studio (Viterbo, 17-19 giugno 1977), Roma, Bulzoni, Centro di studi sul teatro medioevale e rinascimentale, 1978, pp. 207-258.

DELCORNo Carlo, Note sulla tradizione manoscritta delle prediche volgari di S. Bernardino da Siena, «Archivum Franciscanum Historicum», LXXII, 1980, pp. 90-123.

DelCoRno Carlo, Il parlato dei predicatori. Osservazioni sulla sintassi di Giordano da Pisa, «Lettere Italiane», vol. LII, $\mathrm{n}^{\circ}$ 1, 2000, pp. 3-50. 
Enciclopedia Italiana di scienze, lettere ed arti, a cura dell'Istituto della Enciclopedia Italiana, Roma, s.v. Diatriba, vol. XII, pp. 754-755.

Enciclopedia dell'arte medievale, Roma, Istituto dell'Enciclopedia Italiana, 2002.

KIENZLE Beverly Mayne, Medieval Sermon and Their Performance: Theory and Record, in C. Muessig (a cura di), Preachers, Sermon and Society, Leiden, Brill, 2002.

LAUSBERG Heinrich, Elementi di retorica, Bologna, Il Mulino, 1986.

MENÉndez PidAl Ramón, Poesia juglaresca y origines de las literaturas romanicas. Problemas de historia literaria y cultural, Madrid, Instituto de estudios politicos, 1957.

MohrmanN Christine, Le style oral du «De sacramentis » de saint Ambroise, in Ead., Études sur le latin des chrétiens, t. III, Roma, Edizioni di Storia e Letteratura, 1965.

Mortara Garavelli Bice, Manuale di retorica, Torino, Bompiani, 1988.

Mosetti CASARETto Francesco, Assenza della scena: assenza del teatro?, in Id. (a cura di), La scena assente. Realtà e leggenda sul teatro nel Medioevo, Atti delle II Giornate internazionali interdisciplinari di studio sul Medioevo (Siena, 13-16 giugno 2004), Alessandria, Edizioni dell'Orso, 2006, pp. IX-XVII.

RoMANINI Angiola Maria, L'architettura degli Ordini Mendicanti: nuove prospettive di interpretazione, «Storia della città», vol. III, 1978.

Tosti Salvatore, Di alcuni codici delle prediche di San Bernardino da Siena con un saggio di quelle inedite, «Archivum Franciscanum Historicum», XII, 1919, pp. 187-263.

VENTRONE Paola, La sacra rappresentazione fiorentina, ovvero la predicazione in forma di teatro, in G. Auzzas, G. Baffetti e C. Delcorno (a cura di), Letteratura in forma di sermone. I rapporti tra predicazione e letteratura nei secoli XIII-XVI, Atti del Seminario di studi (Bologna, 15-17 novembre 2001), vol. 60, Firenze, Biblioteca di «Lettere italiane», 2003, pp. 255-280.

\section{NOTE}

1. Si veda fra gli altri M. Baxandall, Painting and Experience in Fifteenth Century Italy: A Primer in the Social History of Pictorial Style, Oxford, Oxford University Press, 1988; P. Ventrone, La sacra rappresentazione fiorentina, ovvero la predicazione in forma di teatro, in G. Auzzas, G. Baffetti e C. Delcorno (a cura di), Letteratura in forma di sermone. I rapporti tra predicazione e letteratura nei secoli XIII-XVI, Atti del Seminario di studi (Bologna, 15-17 novembre 2001), vol.60, Firenze, Biblioteca di «Lettere italiane», 2003, pp.255-280; D. Arasse, Saint Bernardin de Sienne. Entre dévotion et culture : fonctions de l'image religieuse au $\mathrm{XV}^{e}$ siècle, Paris, Hazan, 2014.

2. Per un esame più completo si rinvia alle voci «Architettura», «Domenicani» e «Francescani» dell'Enciclopedia dell'arte medievale, Roma, Istituto dell'Enciclopedia Italiana, 2002; si veda anche A. M. Romanini, L'architettura degli Ordini Mendicanti: nuove prospettive di interpretazione, "Storia della città», vol. III, 1978, pp.5-15 e A.Cadei, È possibile scrivere una storia dell'architettura mendicante? Appunti per l'area padano-veneta in Tommaso da Modena e il suo tempo, Atti del Convegno Internazionale di Studi per il VI Centenario della morte (Treviso, 31 agosto-3 settembre 1979), Treviso, Comitato per le celebrazioni, 1980, pp. 337-362.

3. Si veda N. Ben-Aryeh Debby, The Renaissance Pulpit. Art and Preaching in Tuscany, 1400-1550, Turnhout, Brepols, Late Medieval and Early Modern Studies, vol. 6, 2007.

4. B. M. Kienzle, Medieval Sermon and Their Performance: Theory and Record, in C. Muessig (a cura di), Preachers, Sermon and Society, Leiden, Brill, 2002. 
5. Il carattere implicito del teatro era condizione necessaria alla sua stessa sopravvivenza. La Chiesa, infatti, preoccupata dall'esibizione del corpo che il teatro richiedeva e dal carattere idolatrico delle sue manifestazioni, operò una dura condanna di tutte le forme teatrali. Tuttavia, le istanze antropologiche, culturali e sociali sopravvissero sotto forma di teatro implicito negli altri momenti e luoghi del vivere sociale. Si vedano L. Allegri, Teatro e spettacolo nel Medievo, Roma-Bari, Laterza, 1988; F. Mosetti Casaretto, Assenza della scena: assenza del teatro?, in Id. (a cura di), La scena assente. Realtà e leggenda sul teatro nel Medioevo, Atti delle II Giornate Internazionali Interdisciplinari di Studio sul Medioevo (Siena, 13-16 giugno 2004), Alessandria, Edizioni dell'Orso, 2006, pp. IX-XVII; R. Menéndez Pidal, Poesia juglaresca y origines de las literaturas romanicas. Problemas de historia literaria y cultural, Madrid, Instituto de estudios politicos, 1957; C. Casagrande e S. Vecchio, L'interdizione del giullare nel vocabolario clericale del XII secolo, in Il contributo alla drammaturgia italiana delle origini, Atti del II Convegno di studio (Viterbo, 17-19 giugno 1977), Roma, Bulzoni, Centro di studi sul teatro medioevale e rinascimentale, 1978, pp. 207-258.

6. Le seguenti abbreviazioni saranno utilizzate per fare riferimento alle edizioni dei sermoni di Bernardino da Siena: a) F24: Bernardino da Siena, Le prediche volgari, a cura di C. Cannarozzi, Firenze, Tip. Pacinotti, 1934 (L'edizione si basa sul codice Riccardiano 1264 [R] ma vengono registrate alcune varianti di due altri testimoni: Biblioteca Nazionale Centrale di Firenze, cod. II ii 392 [M] e Conventi Soppressi F 61329 [N]. La descrizione dei codici si trova in S. Tosti, Di alcuni codici delle prediche di San Bernardino da Siena con un saggio di quelle inedite, "Archivum Franciscanum Historicum», XII, 1919, pp. 187-263); b) F25: Bernardino da Siena, Le prediche volgari, a cura di C. Cannarozzi, Pistoia, Tip. Pacinotti, 1940 (L'edizione si basa sul codice Conventi Soppressi D.2.1330 della Biblioteca Nazionale Centrale di Firenze, e sui Riccardiani 1353 e 3209); c) S27: Bernardino da Siena, Prediche volgari sul Campo di Siena, 1427, a cura di C. Delcorno, Milano, Rusconi, 1989 (il ciclo senese del 1427 è trasmesso da 21 testimoni per i quali si rinvia alla Nota al Testo nell'edizione citata). Per un esame più approfondito si rinvia a C. Delcorno, Note sulla tradizione manoscritta delle prediche volgari di S. Bernardino da Siena, "Archivum Franciscanum Historicum», LXXII, 1980, pp. 90-123.

7. Si veda Enciclopedia Italiana di scienze, lettere ed arti, a cura dell'Istituto della Enciclopedia Italiana, Roma, s.v. Diatriba, vol. XII, pp. 754-755.

8. C. Delcorno, Il parlato dei predicatori. Osservazioni sulla sintassi di Giordano da Pisa, «Lettere Italiane», vol. LII, $\mathrm{n}^{\circ}$ 1, 2000, pp. 3-50.

9. Si veda S. Arduini e M. Damiani, Dizionario di Retorica, Covilhā, Labcom Books, 2010.

10. C. Mohrmann, Le style oral du «De sacramentis» de saint Ambroise, in Ead., Études sur le latin des chrétiens, t. III, Roma, Edizioni di Storia e Letteratura, 1965.

11. H. Lausberg, Elementi di retorica, Bologna, Il Mulino, 1986, pp.240-242. Si veda anche B. Mortara Garavelli, Manuale di retorica, Torino, Bompiani, 1988.

12. Ibid.

13. F24, XXI, vol. I, p. 335.

14. F24, I, vol. I, p. 2.

15. F25, XLI, vol. II, p. 367.

16. S27, XXXVIII, vol. II, pp. 1102-1103.

17. F24, VII, vol. I, pp. 99-100.

18. F25, XLVI, vol. III, p. 28.

19. F24, III, vol. I, p. 30.

20. F24, XLIII, vol. II, p. 259.

21. F25, XXXIII, vol. II, pp. 225-226.

22. S27, IV, vol. I, p. 198. La predica è dedicata all'enunciazione dei doveri del pubblico e alla memorizzazione della predica.

23. F24, IV, vol. I, p. 63.

24. F24, IX, vol. I, p. 133.

Cahiers d'études italiennes, 29 | 2019 
25. F25, X, vol. I, pp. 182-183.

26. S27, XL, vol. II, p. 1179.

27. S27, III, vol. I, p. 149.

28. F24, X, vol. I, p. 150 .

29. F24, XXXVII, vol. II, p. 141.

30. F24, III, vol. I, p. 36.

31. Ivi, pp. 36-37.

32. S27, XXXV, vol. II, p. 1033.

33. Ibid.

34. F24, XLII, vol. II, p. 236.

35. Ivi, pp. 236-237.

36. S27, XXXVIII, vol. II, p. 1107.

37. Ivi, pp. 1107-1108.

38. F24, XXVIII, vol. II, p. 11.

39. C. Delcorno, Il parlato dei predicatori, cit., p. 42.

40. S27, III, vol. I, p. 144.

\section{RIASSUNTI}

A partire dagli anni Ottanta del secolo scorso, gli studi sui sermoni medievali si sono arricchiti di un nuovo filone di indagine, volto all'analisi dei testi e dei manoscritti attraverso cui le prediche sono state trasmesse. Da allora i sermoni sono stati oggetto di numerose ricerche che ne hanno messo in risalto il valore di 'atti comunicativi', basati sulla diffusione di una 'parola' destinata ad essere pronunciata e udita. L'interesse per lo studio dei testi ha quindi condotto all'analisi di sermoni inediti e alla scoperta delle note stenografiche (reportationes) registrate dagli ascoltatori, durante l'esecuzione stessa del sermone. Il presente contributo svolge un'analisi del corpus omiletico di Bernardino da Siena (1380-1444) e si propone come scopo l'individuazione di elementi che possono essere considerati 'indicatori', segnali, di una modalità espositiva ampiamente coincidente con il codice comunicativo tipico del mondo teatrale. In particolare, l'analisi si rivolge allo studio della figura retorica della sermocinatio che, date le sue caratteristiche, conferisce movimento e vivacità all'esposizione dando vita a una comunicazione di tipo teatrale in cui la mimesi delle espressioni degli interlocutori svolge un ruolo essenziale ai fini della drammatizzazione del testo.

À partir des années 1980, les études sur les sermons médiévaux se sont tournées vers l'analyse des textes et des manuscrits à travers lesquels ceux-ci ont été transmis. Depuis lors, les sermons ont fait l'objet de nombreuses recherches, qui ont mis en évidence la valeur des actes de communication fondés sur la diffusion d'une parole destinée à être prononcée et entendue. L'intérêt porté à l'étude textuelle a donc conduit à l'analyse de sermons non publiés et à la découverte des notes sténographiques (reportationes) prises par les auditeurs lors de l'énonciation du sermon lui-même. Analysant le corpus homilétique de Bernardin de Sienne (1380-1444), cette contribution vise à identifier les éléments pouvant être considérés comme des « indicateurs » ou des signaux d'un mode d'exposition qui coïncide largement avec le code de communication caractéristique du théâtre. L'analyse porte ici, en particulier, sur l'étude de la figure rhétorique de la sermocinatio : compte tenu de ses caractéristiques, celle-ci donne du mouvement et de la 
vivacité à l'exposition, et aboutit à une communication théâtrale au sein de laquelle la mimésis des expressions des interlocuteurs joue un rôle dramatique essentiel.

Since the last decades of 20th century, a new approach was applied for the analysis of medieval sermons. Recent analysis and investigations have focused on the texts and manuscripts through which the sermons were transmitted. Since then, scholars have highlighted the value of sermons as "acts of communication" conveying a message destined to be pronounced and heard. The interest in the study of the texts has therefore led to the analysis of unpublished sermons and to the discovery of the shorthand notes (reportationes) recorded by the listeners during the execution of the sermon. The present contribution focuses on the relationship between preaching and theatre in the Late Middle Ages analysing Bernardino of Siena's sermons (13801444). The research aims at uncovering embedded theatrical elements which can be considered signals or "indicators" of a delivery mode largely coinciding with the typical communicative code of the theatre. In particular, the analysis turns to the study of the rhetorical figure of the sermocinatio: allowing the author to speak dramatically in the first person for someone else, it helps to dramatize the text, providing the basis for a mimesis and allowing the interlocutor to access the stage and play an essential role for the delivery of the sermon.

\section{INDICE}

Mots-clés : Bernardin de Sienne, sermocinatio, théâtre, sermon, prédication médiévale Parole chiave : Bernardino da Siena, sermocinatio, teatro, sermone, predicazione medievale Keywords : Bernardino of Siena, sermocinatio, theatre, sermon, performance theory, medieval preaching

\section{AUTORE}

\section{VALENTINA BERARDINI}

Chercheuse indépendante. valentina.berardini@gmail.com 